\title{
A propos de Troglobies vivant dans les eaux d'une mine de fer de Lorraine
}

\author{
Par Jean-Paul Henry et Chaude Marvillet ${ }^{1}$ )
}

Avec planche 18 (1)

La prospection des eaux des galeries de la mine de fer Orne-Pauline de Moyeuvre-Grande (Moselle) nous avait permis de faire, à la fin du mois de mars 1966, d'intéressantes et nombreuses récoltes de troglobies du domaine aquatique.

Lors d'une seconde exploration nous avons pu retrouver, au début du mois d'avril 1967, non seulement les 5 espèces rencontrées l'année précédente, mais aussi une sixième.

Les emplacements des récoltes se localisent aux abords de la galerie VIII et de ses prolongements.

Du point de vue géologique, les couches de minerai en exploitation, donc les parties accessibles de la mine, sont situées dans l'étage Aalénien, juste sur le niveau des marnes micacées, au voisinage immédiat de l'étage Bajocien. C'est d'ailleurs dans ce dernier que se rencontrent habituellement les faunes de Crustacés Péracarides aquatiques hypogés de Lorraine et de Bourgogne.

L'aspect des points d'eau rencontrés dans la mine varie énormément: il s'agit, soit de flaques permanentes alimentées périodiquement par ruisselets, soit de flaques alimentées continuellement ou de rigoles de drainage, soit enfin de fortes venues d'eau jaillissant d'éboulis d'anciennes galeries effondrées.

\section{La faune}

La faune de ces eaux souterraines se compose de 5 espèces de Crustacés Péracarides (3 Amphipodes, 2 Isopodes) et d'une espèce de

1) Laboratoire de Biologie Animale et Générale de la Faculté des Sciences de Dijon. 
Planaire, dont quelques unes présentent un intérêt biogéographique certain. Ce sont:

\section{GRUSTACES PERACARIDES}

a) Amphipodes (dét. F. Graf)

\section{Niphargus virei Chevreux}

Cette espèce prospère dans la plupart des ruisselets des galeries. $N$.virei, espèce à vaste répartition géographique, est connue de tous les biotopes souterrains (ruisseaux et lacs des grottes, puits, sources, etc.). Husson (1938) signale sa présence dans plusieurs mines voisines du même bassin.

\section{Niphargus aquilex schellenbergi Karaman}

Cette forme cohabite souvent avec la précédente et semble être la plus abondante. Cette remarque rejoint les observations de Husson, qui constatait que cette sous-espèce était le Niphargus le plus fréquent des mines de Lorraine et le seul connu de la mine Orne à Moyeuvre où cet auteur l'avait signalé en 1939.

$N$.aquilex schellenbergi se rencontre dans les différents biotopes souterrains et sa répartition géographique s'étend sur toute l'Europe centrale.

\section{Niphargus kochianus kochianus Bate}

Nous n'avons capturé ce petit Niphargus qu'au cours de la seconde visite de la mine, en avril 1967. Il se trouvait en eau calme, assez profonde, en compagnie de N.aquilex schellenbergi et de la Planaire dont il sera question plus loin, les autres biotopes n'en ayant livré aucun. Nous n'avons vu que de rares individus (trois capturés).

A notre connaissance, cette forme est nouvelle pour les galeries des mines de Lorraine. Husson l'avait également récolté, mais uniquement dans une mine de fer du Jura, en 1937. Sa présence en Lorraine confirme d'ailleurs sa répartition géographique actuelle qui s'étend de l'Irlande à la Bulgarie, en passant par l'Angleterre, la Belgique, la France et l'Allemagne.

Récemment cette espèce a été rencontrée en très grande abondance à Dijon (Côte d'Or), dans la Fontaine des Suisses (Dussart, Graf et Husson, 1966) et dans divers puits de la ville où elle cohabite souvent avec Asellus cavaticus puteanus Henry (Graf et Straskraba, 1967). 


\section{b) Isopodes}

\section{Asellus cavaticus Leydig}

Nous avons capturé 5 ô adultes au cours de la première visite, dans un ruisselet peu profond d'eau légèrement courante à une température de $11^{\circ} \mathrm{C}$. Tous ces individus se déplacent sur des rhizomorphes d'Agaricinées, abondamment développés dans les eaux de la mine, absolument dans les mêmes conditions écologiques que dans la station classique de la mine de Moutiers (Meurthe-et-Moselle).

La seconde fois, malgré des recherches approfondies, aucun exemplaire n'a pu être retrouvé au même endroit, dont cependant les conditions générales étaient demeurées inchangées. Par contre, trois individus très jeunes, 1 ot et 2 \% , ont pu être récoltés également sur des rhizomorphes, mais au voisinage de Caecosphaeroma burgundum, dans un fort courant d'eau à $12^{\circ} \mathrm{C}$.

Tous ces individus, en particulier les mâles de la première récolte, ont fait l'objet d'une étude systématique minutieuse démontrant qu'ils appartiennent sans aucune ambiguïté à l'espèce A.cavaticus telle qu'elle a été définie par l'un de nous (Henry, 1966). La présence d'A.cavaticus dans cette mine était d'ailleurs prévisible car il peuple des eaux souterraines voisines.

Nous rappellerons que cette espèce présente une répartition géographique assez étendue en Europe, mais il s'avère de plus en plus qu'elle est composée de formes distinctes suivant les régions envisagées.

\section{Caecosphaeroma burgundum Dollfus}

C'est un des éléments les plus remarquables de la faune de Moyeuvre. Cet Isopode troglobie «bourguignon», répandu en Saône-et-Loire, Côte-d'Or, dans l'Yonne, en Haute-Marne, Haute-Saône, n'est pas inconnu des milieux souterrains de Lorraine (Meurthe-et-Moselle, Moselle) puisque l'une de ses stations les plus populeuses se situe à Gorze (Moselle), mais sa découverte à Moyeuvre étend son aire de répartition de $22 \mathrm{~km}$. au nord de sa station la plus septentrionale précédemment connue (Marvillet, 1966).

En 1966, 89 individus ont été récoltés en deux endroits différents de la mine, accrochés aux pierres dans un violent courant d'une eau à $12{ }^{\circ} \mathrm{C}$. jaillissant des éboulis d'anciennes galeries. L'effectif de la récolte se décompose ainsi : 14 couples, 3 q ovigères, 16 q adultes, 34 o adultes et 8 jeunes. 
Au mois d'avril 1967, le niveau des eaux étant beaucoup plus bas que l'année précédente, très pluvieuse, la récolte ne fut que maigre aux deux mêmes points d'eau. La découverte d'un troisième très proche devait amener cependant le nombre d'exemplaires rencontrés à un total voisin de celui de mars 1966, soit 88, répartis comme suit : 15 couples, $2 q$ ovigères, $14 q$ adultes, $42{ }^{\star}$ adultes. Les jeunes, assez abondants parmi les pierres, n'ont pas été récoltés; ils sont de toute façon toujours un peu négligés, ce qui explique leur faible proportion dans les récoltes.

Nous retrouvons ici le fait souvent constaté chez cette espèce d'une plus forte proportion de mâles que de femelles.

Notons le fort pourcentage d'animaux accouplés en cette saison (pl. 18, fig. 2 et 3), ce qui vient confirmer des observations menées depuis trois ans au sujet de la périodicité certaine dans l'activité sexuelle de C.burgundum, nettement plus forte en mars-avril qu'à toute autre époque de l'année. Ceci rejoint les conclusions de Ginet chez Niphargus (1960) et de Henry chez Asellus casaticus (1965).

L'aspect extérieur et certaines particularités dans le comportement des individus de Moyeuvre, par rapport à ceux d'une station type de Bourgogne, comme celle de Bévy par exemple, pouvaient laisser supposer des différences sur le plan anatomique. L'examen des détails d'organisation des pièces buccales et des pléopodes o 1 et 2 n'en a cependant révélé aucune jusqu'à ce jour.

La carapace, y compris les appendices, est entièrement recouverte de concrétions granuleuses dont la couleur rouille-clair est vraisemblablement due à une imprégnation d'hydrate ferrique, ce qui confère à ces animaux un aspect peu net opposé à la transparence des exemplaires de Bévy. Il ne peut s'agir, dans le cas présent, d'un agglutination de déchets ou d'un simple dépôt de substances colorées à l'extérieur de la cuticule comme c'est le cas des individus trouvés quelquefois dans d'autres stations après leur séjour dans des flaques isolées (Graf et Marvillet, 1966) ; les conditions sont tout autres à Moyeuvre, étant donné le fort courant d'eau.

Nous avons d'autre part fréquemment observé, dans nos bacs d'élevage, des animaux provenant de cette station qui présentaient une activité très réduite; il s'agissait surtout de mâles de grande taille. Une fois retournés sur les dos, ils se montraient incapables de s'enrouler, de se redresser ou de s'agripper à un support quelconque. Plusieurs sont morts dans ces conditions. Il suffisait cependant, dans la plupart des cas, de produire dans les bacs un courant d'eau, même de brève durée, pour voir aussitôt les animaux reprendre une activité 
vitale normale, s'enroulant et se déplaçant en s'agrippant au bois mort ou aux feuilles.

S'agit-il d'individus habitués à une forte oxygénation de l'eau dans les conditions naturelles de la mine? Notons que les exemplaires récoltés dans de nombreuses stations de Bourgogne, dans des ruisseaux souterrains à fort courant, supportent parfaitement les mêmes conditions d'élevage.

Une autre particularité des Isopodes de Moyeuvre concerne l'effectif moyen d'œufs ou d'embryons contenus dans la cavité incubatrice de la femelle. D'après Daum (1954) et d'après nos propres observations, celui-ci est de 7 à 8 pour les animaux de Gorze. A Moyeuvre, il est de 10. Cette moyenne élevée a été constatée à partir de 15 q ovigères. Des effectifs de 13, ou même 14 œufs, ne sont pas rares.

Parallèlement, nous avons mené des expériences de croisement entre des mâles de Moyeuvre et des femelles de Gorze et de Bévy, et entre des femelles de Moyeuvre et des mâles de Gorze et de Bévy. Jusqu'à présent, c'est-à-dire un an après le début de ces essais, des accouplements nombreux, suivis de ponte et d'incubation, se sont produits entre les animaux de Bourgogne (Bévy) et de Lorraine (Gorze). Par contre, si quelques accouplements ont bien eu lieu entre les animaux de Bévy ou de Gorze d'une part, et ceux de Moyeuvre d'autre part, ils n'ont jamais abouti à des pontes.

Il est prématuré de tirer des conclusions de ces observations, les phénomènes vitaux de C.burgundum étant trop lents pour apporter en une année des renseignements suffisants. Les élevages et les expériences sont poursuivis.

Signalons enfin que des parasites externes (Ostracodes du genre Sphaeromicola) ont été trouvés sur les animaux de Moyeuvre en petit nombre, alors que jamais ceux de Gorze n'en ont présenté.

\section{TURBELLARIES TRIGLADES}

\section{Dendrocoelum (Dendrocoelides) collini de Beauchamp.}

Cette Planaire blanche et aveugle (pl. 18, fig. 1) constitue le second élément intéressant de la faune aquatique de la mine de Moyeuvre. 8 individus en 1966, puis 4 en 1967, ont été récoltés dans une rigole semi-stagnante à fond limoneux parsemé de pierres recouvertes de concrétions calcaires ferrugineuses. Ces pierres sont même soudées au substrat par l'abondance des dépôts.

Une étude sur coupes sériées et la reconstitution de l'appareil copulateur, base de la systématique de ces Triclades, ont prouvé (Marvillet, 
1967) qu'il s'agissait d'une forme locale de Dendrocoelides collini (de Beauchamp, 1919 et 1932). Cette espèce était connue en Côte-d’Or, dans la Marne, la Meuse, en Haute Marne et en Belgique. La forme de Moyeuvre apporte un jalon intermédiaire dans cette aire de répartition géographique.

\section{Conclusions}

Les eaux souterraines de la mine de fer de Moyeuvre sont particulièrement intéressantes puisque leur faune comprend six espèces de troglobies : trois espèces d'Amphipodes du genre Niphargus, dont $N$. kochianus kochianus non encore signalé en Lorraine, deux Isopodes, Asellus cavaticus et Caecosphaeroma burgundum et enfin une Planaire aveugle, forme locale de Dendrocoelides collini.

En dehors de leur intérèt purement biogéographique, la rencontre de $C$.burgundum et $D$. collini a permis de mettre en évidence pour ces deux espèces des traits anatomiques ou physiologiques originaux.

Nous adressons nos vifs remerciements à Monsieur le Directeur de la mine Orne-Pauline, grâce à l'autorisation duquel ces visites ont été possibles, ainsi qu'à MM. Baurens, chef-géomètre, et Schubetzer, ingénieur, qui se sont intéressés à nos recherches et nous ont guidés dans les galeries souterraines.

\section{RÉSUMÉ}

Deux explorations des galeries de la mine de fer Orne-Pauline à MoyeuvreGrande (Moselle), faites à un an d'intervalle, nous ont permis de récolter chaque fois un matériel varié et abondant $(5$ espèces de Crustacés Péracarides et un Ver) de troglobies aquatiques : 3 espèces de Niphargus, dont une nouvelle pour la Lorraine ( $N$. kochianus kochianus), deux espèces d'Isopodes, Asellus casaticus et Caecosphaeroma burgundum (dont c'est la station la plus septentrionale) et enfin une forme locale de Dendrocoelides collini.

\section{SUMMARY}

Two explorations of the galleries of the Orne-Pauline iron mine at Moyeuvre-Grande (Moselle), made one year apart, resulted in the collection on each occasion of varied and abundant material ( $5 \mathrm{spp}$. of peracarid crustaceans and a worm) of aquatic troglobites: $3 \mathrm{spp}$. of Niphargus, one of which is new to Lorraine (N.kochianus kochianus), 2 spp. of isopods, Asellus cavaticus and Caecosphaeroma burgundum (for which this is the northernmost locality), and finally a local form of Dendrocoelides collini. 


\section{BIBLIOGRAPHIE}

De Beauchamp, P. (1919) - Diagnoses préliminaires de Triclades obscuricoles. Bull. Soc. Zool. France, 44, 243-251.

- (1932) - Turbellariés, Hirudinées, Branchiobdellidés. Arch.Zool. exp. gén., $73,113-380$.

DAum, J. (1954) - Zur Biologie einer Isopodenart unterirdischer Gewässer: Caecosphaeroma burgundum Dollfus. Ann. Univers. Saras., 3, 104-160.

Dussart, B., Graf, F., et Husson, R. (1966) - Les Crustacés du réservoir de la Fontaine des Suisses à Dijon. Intern. J. Speleol., 2, 269-281.

Ginet, R. (1960) - Ecologie, éthologie et biologie de Niphargus (Amphipode Gammaridé hypogé). Ann. Spéléol., 15, 239-376.

Graf, F., et Marvillet, Cl. (1967) - Sur la faculté d'absorption de substances colorées dans la cuticule de Caecosphaeroma burgundum Dollfus, Crustacé Isopode des eaux souterraines. Intern. J. Speleol., 3, in litt.

Graf, F., et Straskraba, M. (1967) - Sur une nouvelle sous-espèce de Niphargus jovanovici Karaman. Intern. J. Speleol., 3, in litt.

Henry, J.-P. (1965) - Contribution à l'étude de la biologie d'Asellus cavaticus Leydig. Intern. J. Speleol., 1, 279-286.

- (1966) - Remarques sur l'espèce Asellus cavaticus Leydig et description d'une sous-espèce nouvelle. Intern. J. Speleol., 2, 29-42.

- (1967) - Un Aselle phréatique en Bourgogne. Bull. Sc. Bourgogne, 24, 265-269.

Husson, R. (1938) - La faune des galeries de mines de l'Est de la France. C. R. 1er Cong. Lor. Soc. sas. Est de la France, 73-78.

- (1939) - Amphipodes des galeries de mines de France. Arch. Zool. exp. gén., 81, 101-111.

Marvillet, Ci. (1966) - La station la plus septentrionale de Caecosphaeroma burgundum Dollfus. Yème Cong. nat. spéléol., Bordeaux, in litt.

- (1967) - Variabilité anatomique et répartition de la Planaire troglobie Dendrocoelum (Dendrocoelides) collini de Beauchamp. C. R. Acad. Sci., Paris, 264, 2512-2515.

\section{EXPLICATION DE LA PLANGHE 18 (1)}

Fig. 1: L'une des Planaires aveugles de la récolte de 1967, vue en face dorsale, agrandie 8 fois; la région antérieure se trouve en haut de la photographie.

On remarque, du bas vers le haut: la région de l'appareil copulateur, apparaissant comme une tache plus sombre, puis le pharynx, les nombreux diverticules des caecums digestifs antérieurs, et enfin la partie tout à fait antérieure correspondant à la fossette adhésive.

L'animal est ici dans un état d'extension presque parfait. 
Fig. 2 et 3 : Caecosphaeroma burgundum accouplés, provenant de Moyeuvre, vus en face ventrale (fig. 2) et en face latérale (fig. 3).

La femelle est constamment plus petite que le mâle. De ce fait, les deux animaux peuvent s'enrouler concentriquement en une seule petite sphère. Le mâle agrippe la femelle par ses péréiopodes 2,3 et 4 .

Sur la figure 3 surtout, on remarque les amas plus clairs des organes jaunes, localisés dans le pléotelson et à l'avant du corps. Ils sont beaucoup moins apparents que chez des individus provenant d'autres stations, du fait de l'opacité relative de la cuticule, signalée dans le texte. 


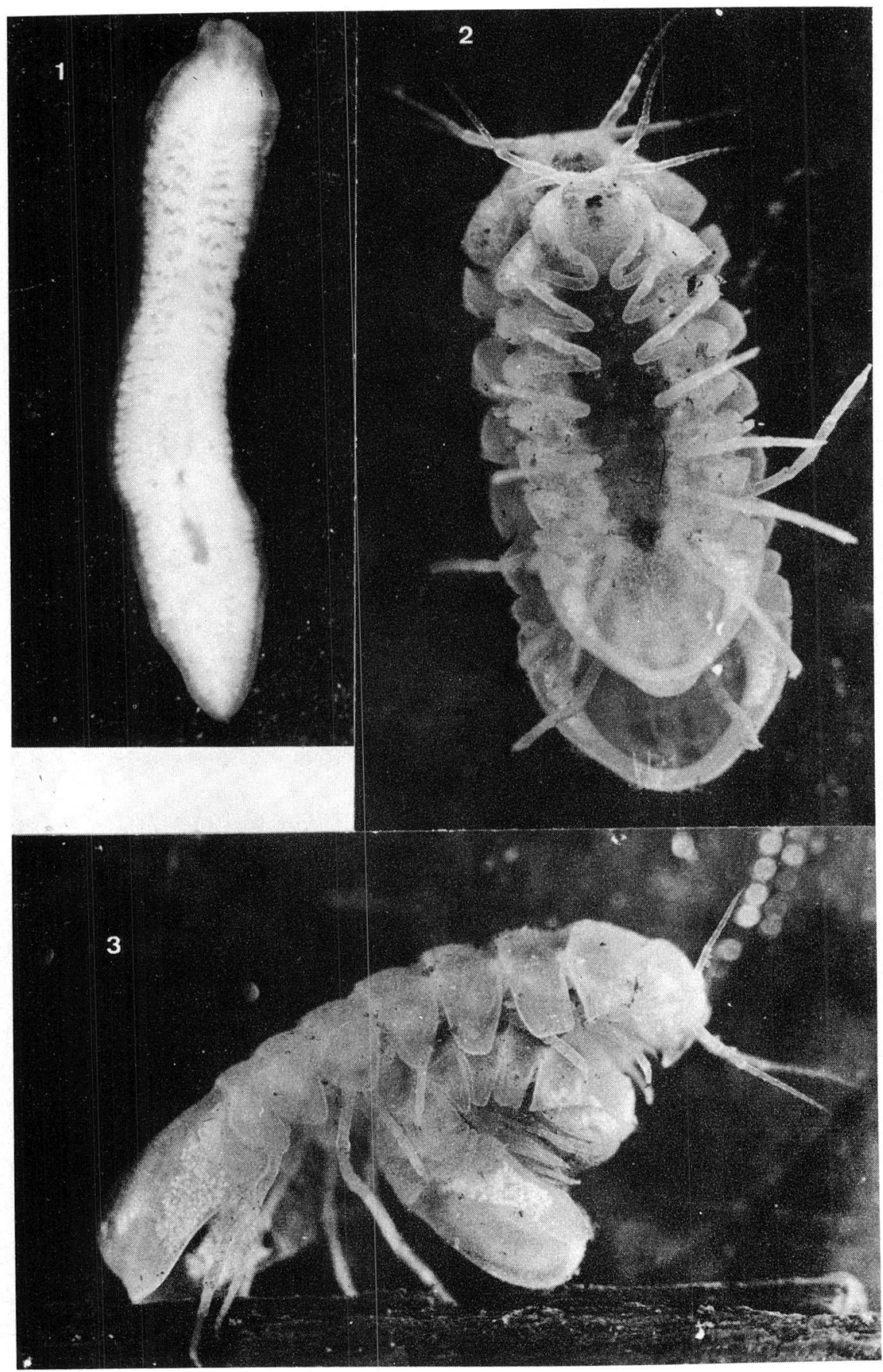

